\title{
A Research on Community Risk Radar with Presetting Forms to Structure Information in Internet of Intelligence
}

\author{
Yifang Leng ${ }^{1,2}$, Chongfu Huang ${ }^{1,2,3, *}$ \\ ${ }^{I}$ State Key Laboratory of Earth Surface Processes and Resources Ecology (Beijing Normal University) \\ Beijing 100875, China \\ ${ }^{2}$ Academy of Disaster Reduction and Emergency Management, Ministry of Civil Affairs \& Ministry of Education \\ the Peoples' Republic of China, Beijing Normal University, Beijing 100875, China \\ 3. Beijing Cazl Technology Service Co., Ltd., Beijing 100088, China
}

Received 22 June 2017

Accepted 3 August 2017

\begin{abstract}
In order to improve the community risk radar in the information gathering stage, the problem of unstructured information processing may be able to result by taking advantages of the internet of intelligence. According to the characteristics of the community risk events, setting the data form of the collecting page in advance can help reduce the proportion of unstructured information. Adding the image upload and download function can help residents building the risk scenarios. In the Risk event assessment stage, coding the PHP web page can achieve the online processing of risk data. In the stage of risk event display, the risk is displayed by the risk radar and risk map. Finally, a community in Beijing is taken as an example for specific description.
\end{abstract}

Keywords: Community, Risk radar, Internet of Intelligences, Structuring information

\section{智联网驱动的社区风险雷达中预设表单式信息结构化研究}

$$
\text { 冷一芳 }{ }^{1,2} \text {, 黄崇福 } 1,2,3, \text {, }
$$

1. 北京师范大学地表过程与资源生态国家重点实验室, 北京 100875

2. 北京师范大学, 民政部/教育部减灾与应急管理研究院, 北京 100875

3. 北京崇安智联科技服务有限责任公司，北京 100088 , 中国

摘 要: 针对现有的风险雷达中非结构化信息的处理问题, 本文提出了基于智联网特点的信息搜集方式, 根据社区风险事件的特点，通过预设搜集页面表单的方式减少非结构化信息所占比例。在风险事件评估阶段， 通过 PHP 网页编码实现风险数据的在线处理; 在风险事件展示阶段, 以风险雷达图辅以风险地图的形式进行 事件展示。此外还增加了风险事件总结阶段, 由管理员对事件的处理状态和处理措施进行更新, 从而使得风 险雷达的数据库成为社区的经验库, 并提供了查询功能, 完成了风险事件排查、匹配以及处理三个环节的统 一。最后，本文以北京海淀区的某社区的试验案例进行了具体说明。

关键词：社区，风险雷达，智联网，信息结构化

* Corresponding author: E-mail: hchongfu@bnu.edu.cn. Post address: Academy of Disaster Reduction and Emergency Management, Beijing Normal University, No.19 Xinjiekouwai Street, Beijing 100875, China

This project was supported by the National Natural Science Foundation of China (41671502), and partly supported by the Beijing Cazl Technology Service Co., Ltd.. 


\section{1.引言}

现代社会的基本单位是社区, 从社区心理学的 角度来说，社区的成员互相之间有许多的关联和共 同认识 ${ }^{[1]}$ 。社区构成了城市, 也是城市管理的基本单 位。二十世纪以来, 社区减灾一直都是国内外采用 的重要减灾战略。1999年, 在 “国际防灾战略”

(ISDR) 中, 联合国已经提出为建设21 世纪更安 全的世界, 需 “从强调政府的作用到重视推进建设 灾害应对能力强的社区” [2], 国内 “十一五” 规划中 有关减灾的重要内容是创建综合减灾示范社区作 ${ }^{[3]}$ 。 无论在哪, 社区灾害风险管理都是减灾和保障可持 续发展的有效手段 ${ }^{[4]}$ 。

在社区, 社区居民从多年的生活中获得了丰富 的应对灾害风险的经验, 此外, 拥有智能可以进行 深入思考的天赋也使得居民能够根据经验感知未来 的风险, 居民通过公共参与的形式加入到社区减灾, 将大大提高社区减灾效率，有利于公众在防灾减灾 中发挥自己独特的作用 ${ }^{[5]}$ 。

风险雷达是能够发现、识别、监测和管理动态 风险的系统, 将风险雷达的理念和社区减灾结合在 一起, 就形成了社区风险雷达 ${ }^{[6]}$ 。社区风险雷达收集 和监测社区内的潜在风险, 它以智联网为驱动, 借 助雷达图的形式动态地展示社区风险事件的变化, 从而为社区管理提供风险监测与预警的功能。在“人 人都是传感器” 的互联网时代, 人机结合的智联网, 正在推动风险分析进入互联网的大数据时代。但是, 目前的社区风险雷达在交叉验证和综合提取, 实现 向结构化信息的转化环节的数据处理仍然依靠人工 操作, 尚无法应对大面积使用带来的大量数据, 不 能达到连续运行的目标。

\section{2. 相关研究现状}

\section{1 智联网简介}

智联网（Internet of Intelligences）是由各种智能 体, 通过互联网形成的, 这一概念最早由黄崇福教 授提出, 目的通过网络集小智慧为大智慧, 群策群 力 $^{[7]}$ 。在数学模型的帮助下, 人们更好地认识世界, 获得更好的生活质量。智联网有三大关键词, 智能 体、网络和模型, 实际上, 智联网是由智能体、网 络、模型组合而成的, 如图1所示。当智联网的智力 水平大于智能体中最高智力水平时, 这是我们期待 的具有集智功能的智联网。

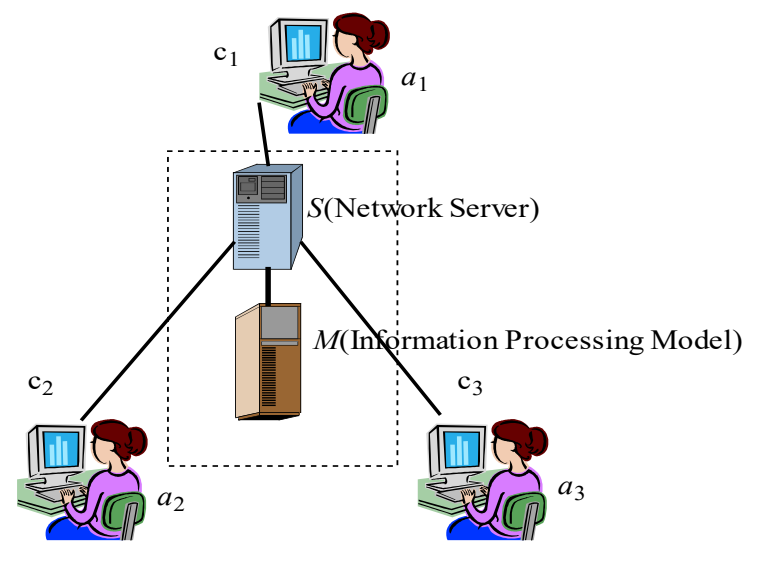

图 1: 智联网示意图

\section{2 智联网应用现状}

目前已有若干智联网平台成功开发并投入使 用, “高考志愿填报智联网服务平台” 是首个研制 成功的智联网演示系统, 可为考生提供有关报考志 愿的参考经验和志愿填报风险评估等功能 ${ }^{[8]}$, “温州 台风灾害风险分析智联网” 是首个投入实用的智联 ${ } \times{ }^{[9]}$, 此外, 基于智联网的灾害风险评估还在城市内

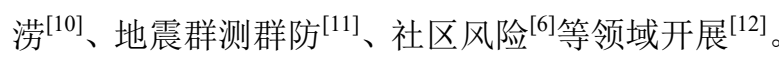

\section{3 风险雷达研究现状}

十九世纪, 科学家在研究蝙蝠夜间飞行原因的 过程中发明了雷达。二十一世纪, 人们开始关注风 险, 能否用雷达来监测风险? 普通的雷达当然不行, 但是在雷达原理的启发下, 人们提出了风险雷达的 概念, 能够发现、识别、监测和管理动态风险的系 统, 被称为风险雷达。

在国内, 风险雷达的概念主要运用在企业风 险监测、化工装备监测、與情监测和社区应急监测 中。在商业银行的风险监测中, “银行风险雷达图” 主要是一种形象化的表达工具, 是将银行的财务状 况、经营成果等信息用各类比率的形式在雷达图上 显示出来, 以便监管人员了解银行运作的风险状况 ${ }^{[13]}$; 在化工装备监测中, 通过篮选装备运行的实时 状态数据识别故障, 获得故障发生的原因和相应补 救措施 ${ }^{[14]}$; 與情监测是互联网对境内外的中文與情 载体（包括新闻网页、论坛、博客、贴吧等）的网 络资源进行采集, 挖掘分析, 把握网民的與论动态、 了解隐患、分析趋势, 并实现话题监控和各种统计 功能 ${ }^{[15]}$ 。

我国是第一个尝试使用智联网驱动技术将风险 雷达应用于社区，用于提高社区应急管理能力的国 
家。与传统商用风险雷达不同的是，智联网驱动的 风险雷达是一个在线系统，而不再是传统风险矩阵 的一种雷达化显示 ${ }^{[16]}$; 与Jovanovic式欧洲风险雷达 不同的是，智联网驱动的风险雷达是一个人机交互 系统; 新兴的风险雷达舆情监测系统不同的是，智 联网驱动的风险雷达不仅仅是对网页海量信息进行 归纳总结的系统。智联网驱动的风险雷达，具有更 强的针对性，提供的风险信息更精细 ${ }^{[17]}$ 。

然而，目前的风险雷达，在信息采集页面的设 计过于简单，导致复式智联网处理社区居民提供的 信息时，在交叉验证和综合提取，实现非结构化信 息向结构化信息的转化环节中，存在问题。由于智 联网获得的风险信息大多是柔性信息，也是非结构 化信息，是人类感知风险的自然语言表述，不能简 单地规范化和数量化, 如自然灾害中的 “洪水齐腰 深”、“䅇泼大雨”等，这类信息在经验信息中占 有较大比例, 而大多数分析模型能够处理的数据是 以规范的和量化的形式表示的 ${ }^{[8]}$, 因而社区风险雷达 的运行还离不开人工的处理环节。

\section{4 社区风险信息结构化研究}

随着计算机网络技术的快速发展，信息以爆炸 式的速度飞速增长, 数据形式变得更加多样化, 信 息的种类也在不断的扩展，其中包括了大量的非结 构化和半结构化数据 ${ }^{[18]}$ 。结构化数据是可以存储在 数据库里, 用二维表的逻辑结构来表达的数据。数 据结构不固定，无法使用关系数据库存储，只能够 以各种类型的文件形式存放的文档、文本文件、图 片、图像、音频和视频等称为非结构化数据 ${ }^{[19]}$ 。介 于两者之间的, 具有结构但结构变化较大, 同时数 据的结构和内容混在一起无法简单区分的数据称为 半结构化数据, 代表性数据为 $\mathrm{xml}$ 文档 ${ }^{[20]}$ 。

非结构化数据存在存储与管理方面的问题, 而 解决这一问题目前通常的思路是通过 “非结构化数 据一半结构化数据一结构化数据” 的方式实现数据 的转换, 从而进行统一有效的管理 ${ }^{[18]}$ 。

但目前的研究大多只是针对于某一类型的文件 展开的,而目前研制的社区风险雷达 ${ }^{[6]}$ 在社区运行过 程中搜集的数据类型包括数字、图片、描述性的大 段文字等，当中的非结构化信息必须在人工进行处 理之后才能进入模型进行计算。当风险雷达在更多 社区中运用时，接收到的大量风险信息显然不能再 依靠人工处理, 因此, 如何将非结构化数据转为结 构化数据, 也是风险雷达面临的几大挑战之一。

\section{3.社区风险信息结构化及社区风险事件}

\section{1 基于智联网的社区风险信息结构化}

智联网获取的信息多为柔性信息, 来源广泛, 包括个体的亲身经历、听闻等。形式上包括零散的 文字、数字，或者是具有逻辑的简短语句，事件的 现场照片等, 是结构化数据和非结构化数据组成的 信息集。其中，为全面描述风险事件，事件描述信 息中包含大段文字的详情描述，属于非结构化信息， 其中的信息提取、分类、真伪判断等工作普通模型 并不能胜任，虽然也可利用复式智联网中人脑的智 能作用参与对这类非结构化经验信息进行处理与分 析 ${ }^{[21]}$, 但效率较慢, 也不方便连接到模型进行运算。

智联网中的智能体具有柔性感知功能 ${ }^{[8]}$, 可以分 辨有关风险事件的干扰信息和片面信息，分析提取 出其中的有效信息, 做出自己的判断, 其原理图如 图2所示。例如, 在面对各类纠纷时, 当事双方往往 各执一词，他们的陈述信息干扰信息和片面信息较 多，而经验老道者往往可以通过二者的描述还原事 件的真实过程, 这一结论的得出即是柔性感知过程。 利用智联网的柔性感知功能, 我们也可以提取出风 险事件的有效信息。

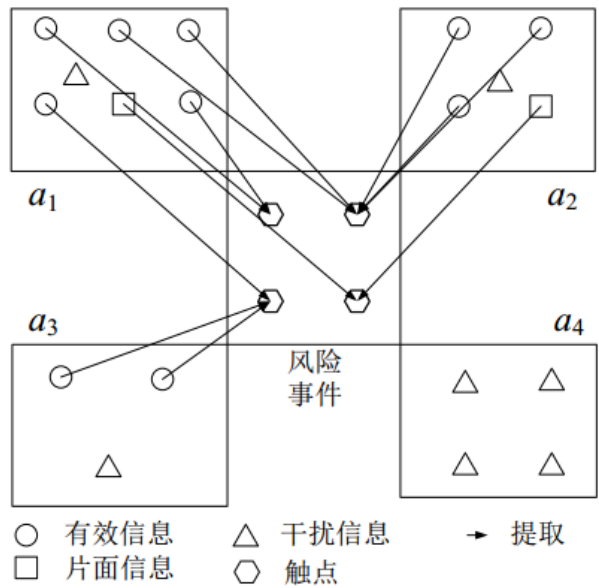

图 2: 柔性感知示意图

为解决风险雷达中非结构化信息转化问题，在 对社区风险事件研究的基础上，通过预设信息搜集 页面的表单，在信息搜集初期，尽量将更多信息从 非结构化的 “事件描述” 字段中剥离, 通过智能体 的柔性感知过程转化为可以直接利用的信息。换言 之, 复杂的详情描述文字信息部分转化为简单的选 择填空形式，以此搜集各类风险问题所需的数据, 剩余部分则依旧依靠复式智能网的人工智能，原理 图见图3。在对社区风险事件深入了解的基础上，如 何通过问题设置来引导和激发智能体的智慧, 就成 为智联网表单设计的一大目标。 


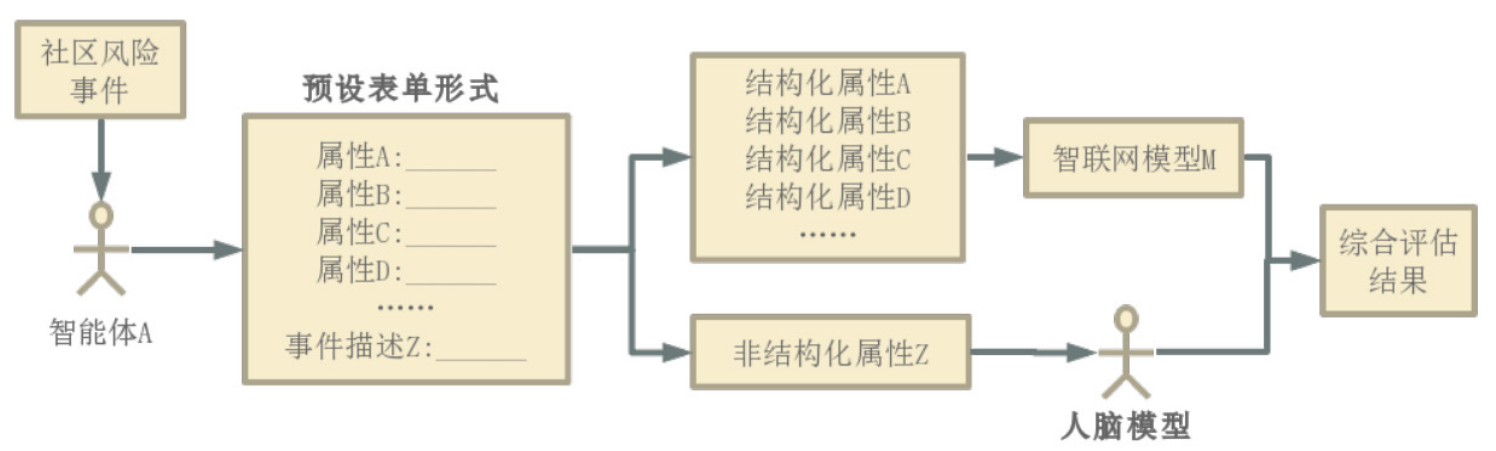

图 3: 预设信息搜集页面表单原理图

\section{2 社区风险事件}

风险是与某种不利事件有关的一种未来情景 [22]。社区风险事件，是发生在社区内部的事件，它 可能会导致社区在未来发生某些不利事件。

社区人口密集，基础设施不足和设备失修老化 等问题往往带来较大后果，具有突发性、威胁性和 不确定性, 透明性和社会性等特点 ${ }^{[23]}$ 。同时, 社区 内发生的风险事件也种类繁多，根据文献阅读和前 期在若干社区的调研情况，依据社区风险事件的不 同属性进行分类, 将有利于风险分析阶段模型对信 息的处理。因此, 根据事件的类型, 事件的发展速 度, 事件的处理方式, 社区风险事件将分为若干类, 按事件类型分类的结果见表1。

表 1：社区风险事件分类表

\begin{tabular}{|c|c|c|c|c|c|c|}
\hline 编 & 事件 & & & 具体 & & \\
\hline 号 & 类型 & & & 分类 & & \\
\hline \multirow{2}{*}{1} & 自然 & 暴雨 & 大风 & 冰雹 & 高温 & 其 \\
\hline & 灾害 & 积水 & 雷电 & 雪灾 & 热害 & 他 \\
\hline \multirow{2}{*}{2} & 火情 & 危化 & 消防 & 燃气 & 触电 & 其 \\
\hline & 隐患 & 品等 & 设备 & 泄漏 & 危险 & 他 \\
\hline \multirow{2}{*}{3} & 治安 & 交通 & 可疑 & 盗窃 & 暴力 & 其 \\
\hline & 交通 & 堵塞 & 人员 & 许骗 & 事件 & 他 \\
\hline \multirow{2}{*}{4} & 设备 & 路面 & 电力 & 通讯 & 其他 & 其 \\
\hline & 隐患 & 损坏 & 设备 & 设备 & 设备 & 他 \\
\hline \multirow{2}{*}{5} & 公共 & 水质 & 垃圾 & 食品 & 疾病 & 其 \\
\hline & 卫生 & 异常 & 处理 & 卫生 & 传播 & 他 \\
\hline \multirow{2}{*}{6} & 居民 & 老年 & 居民 & 装修 & 谣言 & 其 \\
\hline & 活动 & 救助 & 纠纷 & 干扰 & 传播 & 他 \\
\hline
\end{tabular}

\section{4. 社区风险雷达设计与比较}

\section{1 社区风险雷达设计}

根据社区风险雷达的工作原理, 以及社区风险 事件的特点，风险雷达由以下几个层面组成：信息 提交层，包括事件提交页面和事件总结页面; 风险
分析层，包括事件评价页面和风险评估页面；反馈 展示层主要包括事件展示页面, 下面将具体介绍各 页面的设计。

\subsection{1 事件提交页面的表单}

表 2: 事件提交页面表单属性设置

\begin{tabular}{|c|c|c|}
\hline 名称 & 数据类型 & 备注 \\
\hline Pid & $\operatorname{int}(10)$ & 事件编号 \\
\hline Name & $\operatorname{char}(20)$ & 事件类型 \\
\hline Description & $\operatorname{varchar}(200)$ & 事件描述 \\
\hline Lnglat & $\operatorname{char}(20)$ & 位置坐标 \\
\hline location_dis & $\operatorname{char}(20)$ & 位置描述 \\
\hline Start_time & $\operatorname{char}(20)$ & 发生时间 \\
\hline Emerg & $\operatorname{char}(20)$ & 紧急程度 \\
\hline Price & $\operatorname{char}(20)$ & 危险度 \\
\hline Sehnfen & $\operatorname{char}(20)$ & 身份 \\
\hline Image & varchar(40) & 照片上传 \\
\hline Image_dis & Text(200) & 照片详情 \\
\hline Feature2 & Text(20) & 事件状态 \\
\hline Feature3 & Text(20) & 消息来源 \\
\hline Advise & Text(20) & 建议措施 \\
\hline Supply2 & Text(20) & 补充 \\
\hline Create_at & Timestamp(10) & 提交时间 \\
\hline
\end{tabular}

在事件提交页面，用户需要通过文字、图片等 信息来构建风险情景, 尽可能多地提供风险事件相 关信息，社区其他用户才能在此基础上对风险事件 进行风险评价。为了尽可能多地搜集到风险事件的 相关信息，同时尽量将更多信息从非结构化的 “事 件描述” 字段中剥离, 成为可以直接利用的信息, 部分信息以选择或填空形式展现, 以此搜集各类风 险问题所需的数据, 提交页面表单设置见表2。

通过设置不同的属性信息，使得用户头脑中关 于风险事件的抽象记忆能最大程度的表述出来, 也 使得用户提交的信息能够便于理解和处理。其中, 
事件类型以选择形式出现，是基于社区风险事件分 类表, 由于总结性较强, 往往需要辅以简单的事件 描述。位置坐标通过导入百度地图API来获取经纬度 坐标, 有利于对事件进行精确定位, 以及风险地图 模块的表达，但脱离地理信息系统后的经纬度坐标 如在一般人的头脑中并不能映射到某一地点, 因此, 还需添加位置描述用以补充。事件所在地的照片往 往包含许多柔性信息, 难以用语言描述全面, 因此, 采用照片上传和图片描述简单介绍结合的方式使得 信息既全面又概括。

危害度和紧急程度两项指标用来表征普通居民 眼中的风险事件严重程度。此外, 发生时间、事件 状态、消息来源都是风险事件某一属性的具体挖掘: 事件状态包括解决中和已解决, 针对的是社区内的 突发性事件和渐发性事件，突发性事件很有可能已 经得到相应处理, 此外, 在事件得到具体解决后, 事件状态也将在总结页面被管理员修改为已解决, 从而进入社区经验库; 消息来源包括了眼见和听闻 两种, 有利于风险评价阶段的真伪判定。最后, 补 充部分是为了弥补人为分类导致的柔性信息丢失, 对于表单中的未提事宜, 用户可提交到此属性栏中。

通过信息采集和表单设定，尽可能多的结构化 信息与非结构化信息一起组成了用以描述风险事件 的信息集, 后期的结构化信息的风险量化部分工作 量大大减少，也使得其他社区居民可以更轻松地构 建风险事件的风险情景, 并结合自身经验对其进行 评价。

\subsection{2 事件评价页面的表单}

往往, 离风险事件的发生地越远, 人们对事件 的了解程度越低, 可靠性也不足。因此, 事件评价 页面的用户是社区内的人员, 需要提交的内容包括 可能性、危险度、紧急程度、建议解决方向、类似 事件描述、其他补充和身份信息，页面表单的设置 见表 3 。此阶段第一次运用了复式智联网的交叉提取 原理，用户不仅仅提供信息，还参与了信息的处理。 具体来说，其他用户在作为智能体提供的经验信息 进行评价的同时, 也在利用人脑知识库及人脑的智 能作用对事件的初始信息进行处理与分析，这是成 为了风险雷达风险分析模型的组成之一。

在风险事件的评价中, 参考风险矩阵图的内涵, 用户将根据自身经验, 对事件的可能性、紧急度和 危险度三项指标进行打分, 采用相对值0-10的表示 方法, 用以表征风险事件的风险值。为了尽可能的 获取用户有关风险事件的个人经验, 设置了建议解
表 3: 事件评价页面表单属性设置

\begin{tabular}{|c|c|c|}
\hline 名称 & 数据类型及宽度 & 备注 \\
\hline id & $\operatorname{int}(10)$ & 评价编号 \\
\hline Pid & $\operatorname{int}(10)$ & 事件编号 \\
\hline View_trust & float(10) & 可能性 \\
\hline View_risk & float(10) & 危险度 \\
\hline View_emerg & float(10) & 紧急程度 \\
\hline Viewer_shenfen & $\operatorname{char}(20)$ & 身份 \\
\hline Viewer_desc & Text(200) & 其他补充 \\
\hline Viewer_advise & Text(200) & 建议解决方向 \\
\hline Score & $\operatorname{char}(20)$ & 类似事件描述 \\
\hline
\end{tabular}

决方向和类似事件描述两项属性。建议解决方向的 分类基于社区事件的处理部门，引导个体提供相关 经验, 加速事件的解决进程。类似事件描述是针对 社区的某些共通问题设立的，如社区内有人举报有 可疑人员在某居民楼前停留并做标记，用户通过智 联网回答该问题，并扩大和补充可疑人员在社区的 活动范围, 使得社区类似问题可以尽早暴露, 提高 事件的处理效率。在事件评价的过程中，参与其中 的用户不仅能看见事件提交的详细信息，还能参考 其他用户对本事件做出的评价，分辨其中的干扰信 息和片面信息，分析提取出其中的有效信息，并与 自己的个体经验相融合, 从而完成评价过程, 这一 过程是柔性感知的过程 ${ }^{[8]}$ 。

在复式智联网中，智能体不仅仅提供自身感知 的风险信息, 还对其它智能体提供的风险信息进行 柔性感知与捕获，设参与事件提交的用户集为 $\{\mathrm{A}, \mathrm{B}, \mathrm{C}, \mathrm{D}\}$ ，参与事件评价的用户集分布为 $\{\mathrm{A} 1, \mathrm{~A} 2$, $\mathrm{A} 3 \cdots \cdots\},\{\mathrm{B} 1, \mathrm{~B} 2, \mathrm{~B} 3 \cdots \cdots\},\{\mathrm{C} 1, \mathrm{C} 2, \mathrm{C} 3 \cdots \cdots\}$, $\{\mathrm{D} 1, \mathrm{D} 2, \mathrm{D} 3 \cdots \cdots \cdot$, 将两类用户集的用户之间进行 比对, 将会发现用户 $\mathrm{A}$ 参与了其他事件的评论, 这种 情况即为交叉提取。

\subsection{3 风险评估页面的原理}

在事件评价阶段, 复式智联网的参与用户已经 利用人脑的智能作用参与对事件信息进行初步处理 与分析，提交的位置信息、图片信息、消息来源等 为事件评价阶段用户的二次评估和交叉提取提供了 风险事件的具体信息，在此基础上，获取的三项指 标值在经过虚假信息排查后可进入数学模型进行运 算, 通过对事件可能性进行阈值设定, 在排除掉某 些虚假信息后, 采用包括信息扩散在内的数学模型 进行风险评估，其中，风险信息扩散技术是用来弥 补小样本的不完备缺憾, 将样本转化为模糊样本, 
通过PHP编程语言实现了信息扩散技术的模糊样本 优化算法，扩散方法定义如下:

令 $x$ 为区间 $[0,1]$ 的随机样本，使用正态扩散函 数将 $x i$ 变为模糊集 ${ }^{[7]}$, 利用公式 (1-4) 完成信息扩 散计算。

$$
\begin{gathered}
\mu_{x_{i}}(u)=\exp \left(-\frac{\left(x_{i}-u\right)^{2}}{2 h^{2}}\right), \quad u \in[0,1] \\
f(u)=\sum_{i=1}^{n} \mu_{x_{i}}(u), \quad u \in[0,1] . \\
f\left(u_{0}\right)=\sup _{u \in[0,1]} f(u) \\
\operatorname{diffusing}\left(x_{1}, x_{2}, \ldots, x_{i}, \ldots, x_{n}\right)=u_{0}
\end{gathered}
$$

在对三项指标进行信息扩散计算后，风险值也 可以计算出来。考虑到风险是在特点时间和空间范 围内的潜在损失大小及发生的可能性，将以风险源 的发生概率和承灾体的暴露性和脆弱性表征风险 ${ }^{[24]}$, 可能性指标代表了风险源的发生概率，考虑到社区 风险事件的突发性和威胁性, 将危险度和紧急程度 两项指标代表承灾体的暴露性和脆弱性。用 $\mathrm{P}$ 代表可 能性, $\mathrm{E}$ 代表暴露性和脆弱性, 风险评估值 $\mathrm{R}$ 的模型 可形式化表示为式 (5):

$$
\mathrm{R}=\mathrm{P} \bigcirc \mathrm{E}
$$

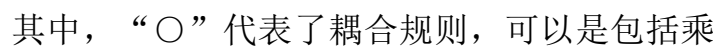
法运算等在内的数学公式 ${ }^{[25]}$ 。考虑到风险与特定时 间相关, 具有动态性, 随着时间的推移, P、E的变 化将导致风险表征值R也发生变化, 因此, 评估过程 是不断进行的。

\subsection{4 事件展示页面的设计}

风险时时刻刻都在发生变化, 随着时间的推移, 不断有智能体加入到风险评估中，提供自己的经验 信息, 此时发生变化的不仅仅是风险评估的结果, 还包括可能风险事件本身。当事件得到及时解决, 事件状态从待处理变为已解决时, 应将该事件从待 评价列表中撤下, 使得居民的注意力转移到新的风 险事件上。因此, 为了更好的反映事件的变化, 评 估结果是实时更新并展示出来的。展示页面包括首 页的风险雷达图和风险地图两个页面。

风险雷达图即图 4 , 社区风险雷达以智联网为驱 动, 获得不断更新的风险评估结果, 并在, 风险雷
达图中表现出来。然而社区风险事件多发，过多的 风险事件展示在风险雷达图上，不仅可能导致社区 内发生恐慌, 还可能充塞雷达图, 分散居民的注意 力，导致居民对风险事件关注度下降，忽视某些风 险事件。因此, 首页风险雷达图中风险事件的显示 仍然离不开系统管理员的审核。

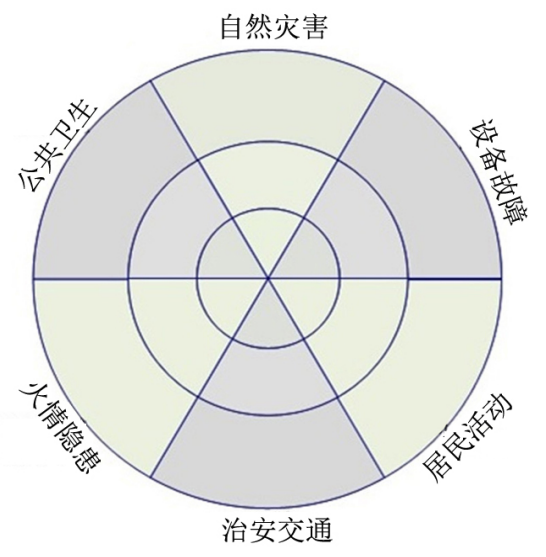

图 4 : 风险雷达图

风险雷达图是基于事件分类进行展示，除此之 外, 还有基于地理信息的风险地图展示页面。在风 险地图中，各风险事件依据经纬度坐标展示在百度 地图插件中，以圆形表示，圆形的颜色代表风险事 件的风险值大小，见图10。在长期使用后，数据经 过整理, 风险地图可以升级为社区灾害风险地图。

\subsection{5 事件展示页面的设计}

此阶段第二次运用了复式智联网的原理, 主要 用户为社区风险雷达管理者，可以是居委会工作人 员，业主委员会成员，或是经社区认证的热心居民。 在参考事件评价阶段获取的若干个体经验后，事件 得到相应处理, 为了促进社区风险雷达数据库建设 成为社区的经验库, 用户需要在事件总结页面进行 总结, 页面表单的设置见表4。

表4: 事件总结页面表单属性设置

\begin{tabular}{lll}
\hline 名称 & 数据类型及宽度 & 备注 \\
\hline Pid & $\operatorname{int}(10)$ & 事件编号 \\
Feature2 & Text(20) & 事件状态 \\
S1 & Text(20) & 实际解决措施 \\
S1 & Text(200) & 措施详情 \\
S3 & Text(200) & 如何规避风险 \\
\hline
\end{tabular}


总结页面主要包括四项内容：事件状态、解决 措施分类、措施详情、如何规避风险。事件状态需 从待处理改为已解决或解决中, 从而使得事件从待 评价列表以及风险雷达图上撤下。公布事件的解决 措施分类以及措施详情将为社区提供更为丰富的应 对经验，减少未来遇到同样问题的应对时间。最后， 利用人脑的智能作用对事件的相关信息进行柔性感 知的处理与分析, 参考其他智能体的经验, 总结出 规避风险的办法，避免风险事件在未来产生不利后 果。

\section{2 与传统社区风险雷达的比较}

图 5 为社区风险雷达改进前后的工作流程 对比, 与传统社区风险雷达 ${ }^{[6]}$ 相比, 改进后的风险雷 达在工作流程上主要区别包括：风险事件提交、评
价页面的改变, 由于预设表单页面的设计, 新经验 信息集中获取的非结构化信息较少，大部分数据可 以进入模型中直接处理; 模型处理和评估阶段, 通 过 PHP 网页编码实现风险数据的在线处理, 可以得 到实时的评估结果; 反馈展示阶段增加了风险地图 的表达; 事件总结页面的设置, 增加了社区风险事 件的处理终点, 社区经验库的开放使得个体经验成 为社区的共同经验。

\section{5. 案例背景与分析}

\section{1 案例背景}

北京的住宅小区中绝大多数为老旧小区，由多 层住宅楼和平房胡同组成，社区内部问题较多，社 区管理难度也较大。选择老旧小区为实验社区，可 以更快地提高社区风险雷达的实际应用能力。在社

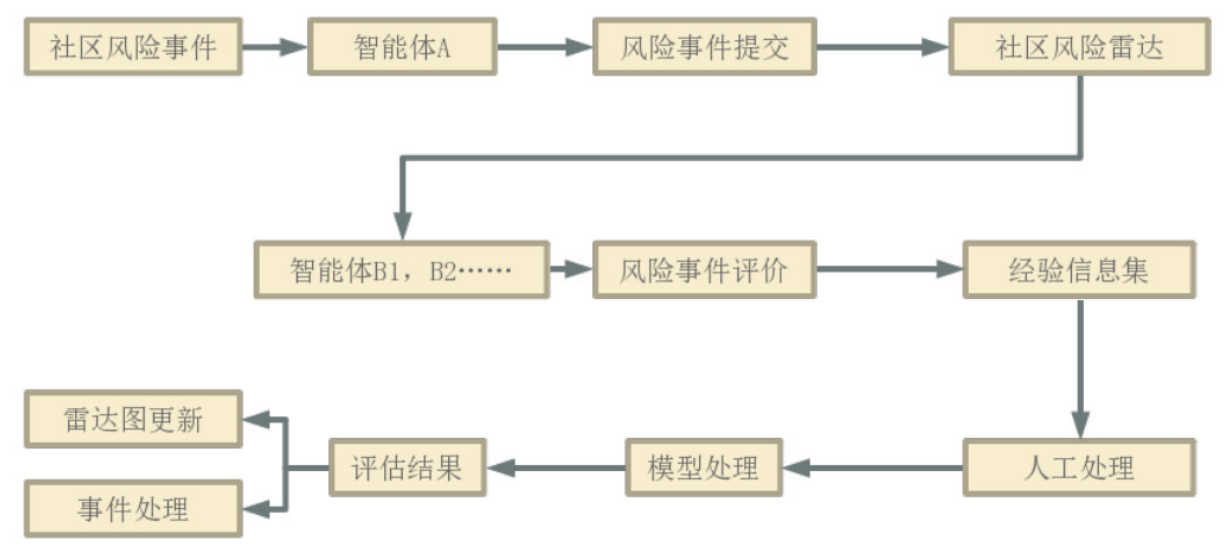

（a）原社区风险雷达工作流程图

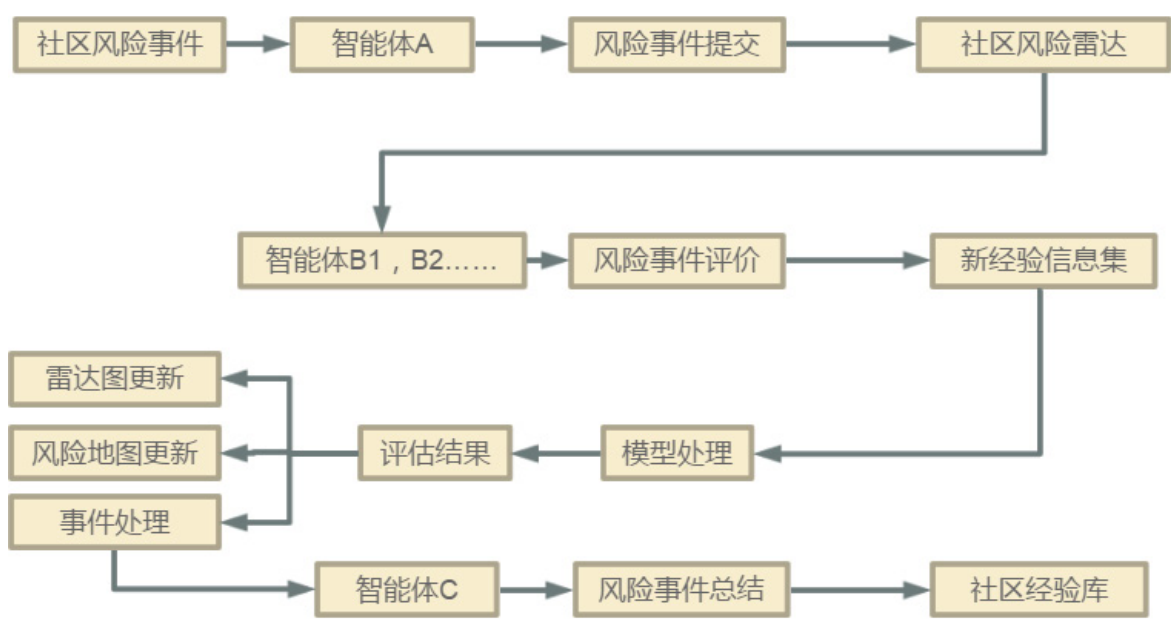

(b) 改进的社区风险雷达工作流程图

图 5: 社区风险雷达改进前后的工作流程对比. 
区居委会的宣传和帮助下，社区居民在社区风险雷 达平台提交和评论社区风险事件，通过交叉提取和 柔性感知进行风险评估，并实时发布至平台，管理 员结合居民的评估意见和实际情况处理风险事件, 并对风险事件进行总结, 完善社区经验库。

实验社区 D 位于北京市海淀区东南部，处于北 下关街道辖区。北下关街道面积约 60.4 万平方米, 辖社区 31 个。实验社区占地 6.6 万平方米, 共有楼 房 19 栋, 平房院落 5 个, 是一个楼院、平房共存的 小区。常住人口户数 1901 户 ${ }^{[26]}$ 。社区地处城乡结 合部, 区内有大型央企, 冷冻食品厂, 学校, 家乐 福超市, 综合性医院, 酒店宾馆等, 居民生活区域 和生产区域并未分离。由于社区建立较早, 是老旧 社区, 社区硬件比较差。除治安问题外, 社区内平 房区安全用火、用气、用电安全是社区管理工作的 重心。社区所在街道己建立有微信公众号平台, “随 手拍文明” 等活动已开展近半年 ${ }^{[27]}$ ，社区居民对于 使用网络平台来反映社区公务事务有较高积极性。

在居委会的宣传和帮助下， 2017 年 3 月 28 日 至 4 月 1 日期间社区风险雷达在 $\mathrm{D}$ 社区进行了试用。 社区居民可以通过电脑或手机登陆社区风险雷达平 台, 提交他们在社区内发现的风险事件。期间共收 集到风险事件 10 起, 收集到事件评论 30 多份。其 中, 关注度最高的是设备隐患中的电线树枝纠缠事 件, 下面将以此社区风险事件进行具体说明。

\section{2 案例分析}

电线树枝纠缠事件发生在 D 社区丙四号院外, 事件类型属于设备隐患。院内大树的枝干伸出院门, 与院外道路一侧的电力线纠缠在一起, 树枝与电线 之间的摩擦将加速电线外壳的磨损速度，在强风、 暴雨、冰雹等灾害性天气出现时, 树枝的存在对电 力线的安全威胁更加严重, 容易给过往行人和车辆 带来灾难性后果。

本起风险事件中提交者提交信息见图 6, 并上传 了图片加以佐证, 点击照片上传右侧链接, 即可下 载图片进行查看, 风险事件图片见图 7。

共有 8 位居民参与了事件评价, 通过在社区风 险雷达评价管理页面输入事件编号, 可查询本次风 险事件的全部评价，见图 8。

风险事件经过评价阶段的柔性感知和交叉提 取, 在风险评估阶段不断更新。随着参与人数的增 加, 数据样本不断扩大, 风险值也越来越接近真实 值，具体地，根据定义的风险计算公式得到的风险 表征值变化如表 5 。
本次社区风险事件经管理员审核后，出现在首 页风险雷达图上, 随着评论人数的增加, 雷达图中 图标的位置也发生相应变化, 表征着风险事件的风 险值变化, 见图 9。

风险事件发生前后, 风险地图的变化如图所示, 图 10 为发生后, 发生地用圆圈圈出。

\section{社区风险雷达}

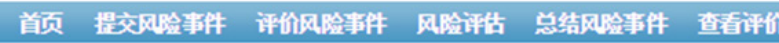
Home » Radar Viewers » Create

评价风险事件

\begin{tabular}{|c|c|}
\hline 事件编号 & 59 \\
\hline 事件详细类型 & 设备隐患电力设备 \\
\hline 事件描述 & 院内大树的树枝伸出墙外, 压着路边的电线 \\
\hline 位置㩲述 & 丙4号院外 \\
\hline \multicolumn{2}{|c|}{ 发生时间 } \\
\hline 照片上传 & IMG 20170401 091031.jpg \\
\hline 照片描述 & 树枝压着电线 \\
\hline 危脸度 & 8.00 \\
\hline 紧急程度 & 5.00 \\
\hline 建议措施 & 很担心会属电, 希望派人修梖树枝 \\
\hline \multicolumn{2}{|l|}{ 补充 } \\
\hline 事件犾态 & 待处理 \\
\hline 消息来源 & 亲眼所见 \\
\hline 提交时间 & 2017-04-01 09:45:10 \\
\hline 隹的身份 & 丙4号院居民 \\
\hline
\end{tabular}

图 6 ：风险事件提交信息

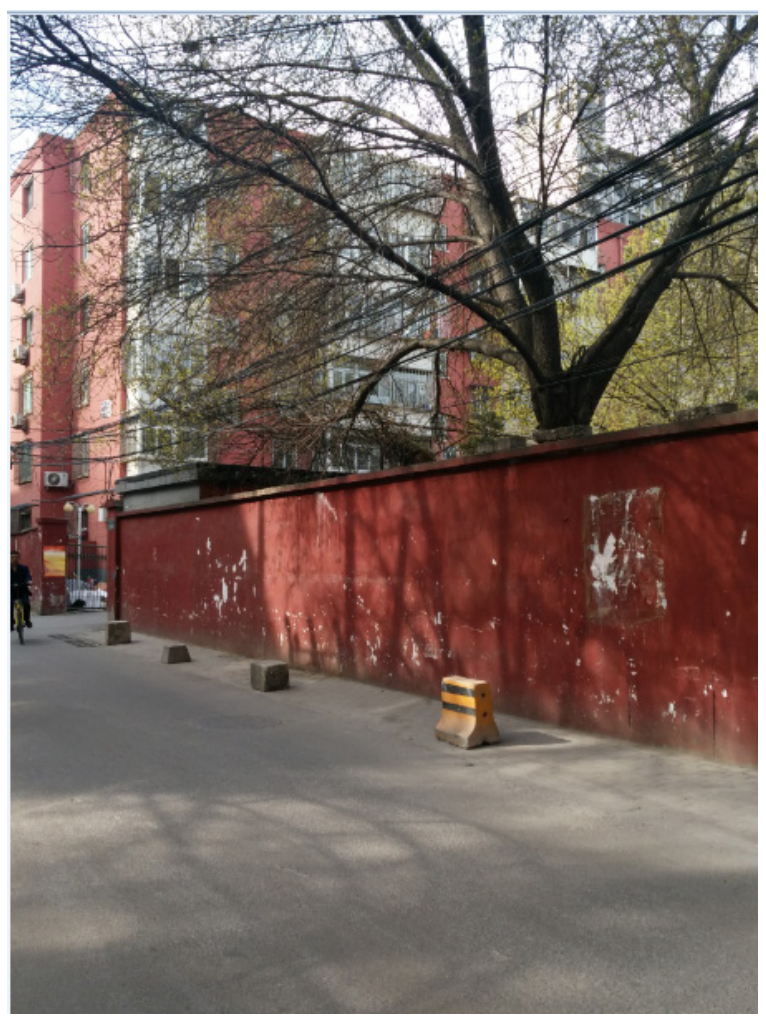

图 7 : 风险事件现场图 


\section{社区风险雷达}

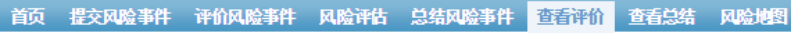

Home ” Radar Viewers » Manage

评价管理页面

1.输入事件编号, 可查洵到风险事件相关评论, 点击放大镜图标', 可查看评价详情 2.占击"凤崄事件列表",可查涧到风险事件初始信息

\section{风险事件提交列表}

Advanced Search

\begin{tabular}{|c|c|c|c|c|c|c|c|}
\hline 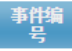 & 评售 & 可能性 & 色踰度 & 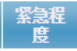 & 筧射身 & 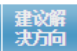 & 其伤补充 \\
\hline \multicolumn{8}{|l|}{59} \\
\hline 59 & 31 & 7.00 & 8 & 3 & 丙4居民 & $\begin{array}{l}\text { 社区内部 } \\
\text { 协调 }\end{array}$ & 怕电线和树枝摩摖多了把电线给烧 \\
\hline 59 & 32 & 8.00 & 8 & 7 & 院内居民 & $\begin{array}{l}\text { 社区内部 } \\
\text { 协调 }\end{array}$ & $\begin{array}{l}\text { 树技离电线确实太近了, 北京风又 } \\
\text { 大, 比较色险 }\end{array}$ \\
\hline 59 & 33 & 3.00 & 4 & 3 & 社区居民 & $\begin{array}{l}\text { 社区内部 } \\
\text { 协调 }\end{array}$ & 感觉这个威胁不算大 \\
\hline 59 & 34 & 9.00 & 8 & 5 & $\begin{array}{l}\text { 甲133院 } \\
\text { 居民 }\end{array}$ & $\begin{array}{l}\text { 上报分管 } \\
\text { 部门 }\end{array}$ & $\begin{array}{l}\text { 怕夏天树叶长起来了更危险, 早点 } \\
\text { 修留吧 }\end{array}$ \\
\hline 59 & 35 & 9.00 & 9 & 5 & 居民 & $\begin{array}{l}\text { 上报分管 } \\
\text { 部门 }\end{array}$ & $\begin{array}{l}\text { 早点派人来修塑下吧, 我们自己又 } \\
\text { 不动动手, 着总是怕㤛的 }\end{array}$ \\
\hline 59 & 36 & 9.00 & 9 & 8 & 居民 & $\begin{array}{l}\text { 上报分管 } \\
\text { 部门 }\end{array}$ & 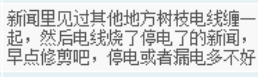 \\
\hline 59 & 38 & 8.00 & 5 & 9 & 附近居民 & $\begin{array}{l}\text { 社区内部 } \\
\text { 协调 }\end{array}$ & 刮风的时候特别怕电线刮坏了 \\
\hline 59 & 39 & 8.00 & 8 & 6 & $\begin{array}{l}\text { 甲133院 } \\
\text { 居民 }\end{array}$ & $\begin{array}{l}\text { 上报分管 } \\
\text { 部门 }\end{array}$ & 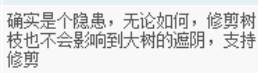 \\
\hline
\end{tabular}

图 8: 风险事件评价查询结果

表 5 : 风险表征值变化

\begin{tabular}{|c|c|c|c|c|}
\hline 参与人数 & 危险度 & 可能性 & 紧急程度 & 风险值 \\
\hline 1 & 8 & 7 & 3 & 6.25 \\
\hline 2 & 8 & 7.5 & 5 & 7 \\
\hline 3 & 6.67 & 6 & 4.33 & 5.75 \\
\hline 4 & 7 & 6.75 & 4.5 & 6.25 \\
\hline 5 & 6.70 & 6.24 & 44.91 & 6.02 \\
\hline 6 & 6.97 & 6.54 & 5.38 & 6.36 \\
\hline 7 & 6.92 & 6.78 & 5.88 & 6.59 \\
\hline 8 & 7.07 & 6.99 & 5.88 & 6.73 \\
\hline
\end{tabular}
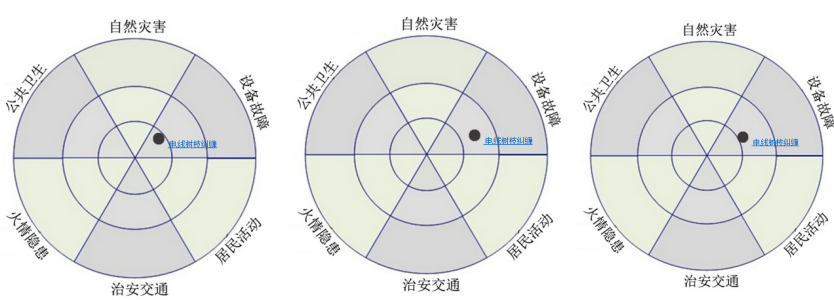

图 9: 风险雷达图变化

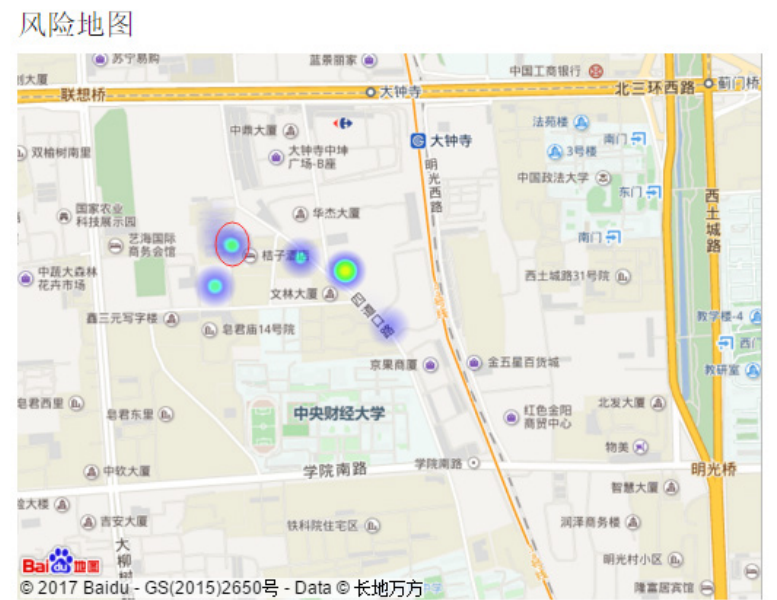

图 10 ：某风险事件发生后

由于树枝修剪工作专业性较强，且可能存在触 电风险, 社区居民无法自行处理, 社区内也无物业 管理公司负责此项事务。在核实情况后，管理员将 事件上报至本区园林部门, 请绿化队工作人员携带 专业工具完成修剪工作，并在总结页面提交事件处 理方案，并将事件状态改为 “处理中”，该事件处理 进度可在列表中查询到, 见图 11, 输入事件编号“59” 即可。修剪工作完成后, 管理员可将事件状态修改 为 “已解决”。

\section{社区风险雷达}

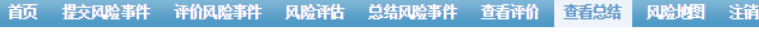 Home " Radar Sums » Manage \\ 事件总结列表 \\ 点击“族大镜"图标, 可查着总结详情 Advanced Search

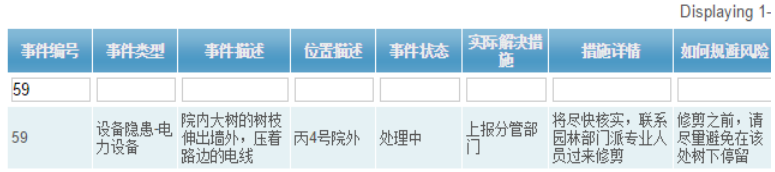

图 11：事件总结信息查询

本次风险事件过程中搜集到的经验信息也已进 入数据库, 可以在 “查看评价”、“查看总结” 模块 查询到具体信息, 即历史风险事件匹配过程, 在 “事 件描述” 下方的文本框内输入 “电线”, 也可以查询 到本起事件。通过事件的积累丰富社区的风险事件 应对经验库，将避免重复工作，大大提高工作效率。

\section{6. 结论}

本文以智联网驱动的社区风险雷达为研究对 象, 通过尝试深入了解社区风险事件以及社区居民 的风险意识和表述习惯，研制一系列柔性感知过程 
中使用的表单, 实现数据的在线处理功能, 形成自 动运行的复式智联网, 同时利用智联网数据库实现 了社区经验库的功能, 持续驱动社区风险雷达, 完 成社区风险雷达设计中隐患排查、历史事件匹配以 及事件处理的目标。针对现有的风险雷达中非结构 化信息的处理问题，提出基于智联网特点的信息结 构化搜集方式，针对社区风险事件的特点，通过预 设搜集页面表单的方式减少非结构化信息所占比 例, 并具体介绍了社区风险雷达中各个环节的设计。 通过实验社区的案例, 展示了社区风险雷达的试用 情况、评估过程及评估结果等, 验证了平台的实用 性。实验过程中，社区居民通过社区风险雷达反映 问题的愿望较强烈, 社区工作人员则希望能够通过 社区风险雷达改善社区环境, 减轻工作量。

\section{参考文献}

[1] 刘视湘. 社区心理学. 开明出版社,2013:60-60.

[2] 陈徐东. 社区防灾减灾保障机制的创新. 城市与减 灾,2010(6):5-7.

[3] 卫敏丽. 解读《国家综合减灾“十一五”规划》. Disaster Reduction in China, 2007,(9):19-22.

[4] 陈容,崔鹏. 社区灾害风险管理现状与展望. 灾害 学,2013,28(1):133-137.

[5] 冷一芳,黄崇福. 社区应急管理风险雷达中预设表单 式信息结构化研究初探. 大数据时代的风险分析和 危机反应----中国灾害防御协会风险分析专业委员会 第七届年会. 2016,11:890-895.

[6] 吴粀. 智联网驱动的在线风险雷达在在社区应急管 理中的应用研究. 北京:北京师范大学,2015.

[7] 黄崇福. 自然灾害风险分析与管理. 科学出版社, 2012.

[8] 艾福利,黄崇福,王蔚丹. 高考志愿填报风险评估智联 网服务平台. 风险分析和危机反应的创新理论和方 法一一国灾害防御协会风险分析专业委员会第五 届年会论文集,2012.

[9] 黄崇福. 自然灾害风险评价:理论与实践. 北京:科学 出版社, 2005 .

[10] 郭君,艾福利,蒋卫国,等. 自然灾害风险分析智联网 中的柔性地理信息及其应用。风险分析和危机反应
中的信息技术--中国灾害防御协会风险分析专业委 员会年会.2014.

[11] 王蔚丹,黄崇福,曾凡雷. 地震群测群防智联网中双层 虚假问题的研究. 风险分析和危机反应中的信息技 术--中国灾害防御协会风险分析专业委员会年会. 2014.

[12] 苏妩. 信息收集去中心化在风险评估中的应用 ---海 洋环境风险管理智联网平台研究. 北京: 北京师范大 学,2016.

[13] 周晓阳. 改进的雷达图分析法在我国商业银行风险 监测中的应用。今日财富: 金融发展与监 管,2010(6):59-64.

[14] 中国专利发布公告.

[15] http://epub.sipo.gov.cn/pam.action

[16] 苏博. 太原供电公司网络舆情监测系统的设计和实 现. 华北电力大学,2014.

[17] 黄崇福. 自然灾害动态风险分析基本原理的探讨,灾 害学,Vol.30, No.2, (2015), 1-7,

[18] 吴粀,艾福利,郑建军,黄崇福. 智联网驱动的风险雷 达实现探讨 $[\mathrm{A}]$. 风险分析和危机反应中的信息技术 --中国灾害防御协会风险分析专业委员会第六届年 会论文集.中国灾害防御协会风险分析专业委员 会,2014,6:468-473.

[19] 万里鹏. 非结构化到结构化数据转换的研究与实现. 西南交通大学,2013.

[20] 徐宗本,张讲社. 基于认知的非结构化信息处理:现状 与趋势. 中国基础科学,2007,(6):53-55.

[21] 许学标,顾宁. 半结构化数据模型及查询语言. 计算 机研究与发展, 1998,(10):896-901.

[22] 艾福利. 自然灾害风险分析智联网服务平台构建与 应用研究. 北京:北京师范大学,2013.

[23] 黄崇福. 自然灾害风险评价理论与实践. 科学出版 社, 2006.

[24] 乔苹. 我国城市公共危机治理中的公民参与机制研 究. 首都经济贸易大学, 2013.

[25] 马保成. 自然灾害风险定义及其表征方法。灾害 学,2015,(3):16-20.

[26] 黄崇福,郭君,艾福利,等.洪涝灾害风险分析的基本 范式及其应用. 自然灾害学报,2013,(4):13-25.

[27] 社区简介. http://mp.weixin.qq.com/s/OyA07YvR_8oco6y0O_FI $3 \mathrm{w}$

[28] “你来拍,我来奖”活动网址. https://sanwen8.cn/p/556CBwf.html 